\title{
Measurement of quality of life using EQ-5D in patients on prolonged mechanical ventilation: comparison of patients, family caregivers, and nurses
}

\author{
Mei-Chuan Hung • Yuan-Horng Yan • Po-Sheng Fan • Ming-Shian Lin • \\ Cheng-Ren Chen • Lu-Cheng Kuo • Chong-Jen Yu • Grace Yao • \\ Ching-Lin Hsieh · Jung-Der Wang
}

Accepted: 25 February 2010/Published online: 12 March 2010

(C) Springer Science+Business Media B.V. 2010

\begin{abstract}
Purpose This study reports how QOL (quality of life) assessments differ between patients on prolonged mechanical ventilation (PMV) and their proxies (family caregivers and nurses).

Methods We enrolled consecutive subjects on PMV for more than 21 days from five institutions. We conducted QOL assessments using the Taiwanese version of the EQ5D in face-to-face interviews. Direct caregivers (family members and nurses) also completed the EQ-5D from the patient's point of view.

Results For 55 of the 142 enrolled patients who were able to assess their QOL, we recruited 44 patient-family
\end{abstract}

M.-C. Hung · J.-D. Wang ( $ه)$

Institute of Occupational Medicine and Industrial Hygiene,

College of Public Health, National Taiwan University, Rm. 719,

No 17, Xuzhou Rd, Taipei, Taiwan

e-mail: jdwang@ntu.edu.tw

Y.-H. Yan · P.-S. Fan · M.-S. Lin · C.-R. Chen

Department of Internal Medicine, Chia-Yi Christian Hospital,

Chiayi, Taiwan

L.-C. Kuo $\cdot$ C.-J. Yu $\cdot$ J.-D. Wang

Department of Internal Medicine, National Taiwan University

Hospital, Taipei, Taiwan

G. Yao

Department of Psychology, National Taiwan University,

Taipei, Taiwan

\section{C.-L. Hsieh}

Department of Occupational Therapy, National Taiwan

University, Taipei, Taiwan

J.-D. Wang

Department of Environmental and Occupational Medicine,

National Taiwan University Hospital, Taipei, Taiwan caregiver pairs, 53 patient-nurse pairs, and 42 family caregiver-nurse pairs. There were 81 family caregiver-nurse pairs out of 87 patients with poor cognition. The agreement between patient-family caregiver pairs was generally higher than that of patient-nurse pairs. As the proportions of exact agreement between family caregivers and nurses for patients with poor cognition were 98-99\% for observable dimensions of mobility, self-care, and usual activities, they lead to a minimal difference in the final values.

Conclusions QOL assessments from family caregivers agreed more closely with patients than did those from nurses using EQ-5D evaluations for patients with clear cognition, but either proxy was acceptable for rating PMV patients with poor cognition.

Keywords Prolonged mechanical ventilation . Proxy $\cdot$ Quality of life $\cdot$ EQ-5D $\cdot$ Utility

$\begin{array}{ll}\text { Abbreviations } \\ \text { PMV } & \text { Prolonged mechanical ventilation } \\ \text { QO } & \text { Quality of life } \\ \text { ICU } & \text { Intensive care unit } \\ \text { RCC } & \text { Respiratory care center } \\ \text { RCW } & \text { Respiratory care ward } \\ \text { IRB } & \text { Institutional review board } \\ \text { MMSE } & \text { Mini-mental status examination } \\ \text { EQ-5D } & \text { EuroQol five-dimensional } \\ \text { ICC } & \text { Intra-class correlation coefficient }\end{array}$

Introduction

Patients who require prolonged mechanical ventilation (PMV) are rapidly increasing in number, as the improved 
quality of care in ICUs (intensive care units) has resulted in long-term survival for many patients $[1,2]$. However, these patients often require continued respiratory care after transfer to a rehabilitation facility, skilled nursing facility, or home care, creating financial burdens for the insurance system and/or the patients' families [1, 3]. Several studies have reported [1, 4-6], however, that survivors often feel their quality of life (QOL) to be acceptable.

The National Health Insurance (NHI) of Taiwan uses a system of comprehensive coverage for various health care services, including maintenance hemodialysis and chronic respiratory care. The NHI was first established in 1995 and has been extended to cover over $99 \%$ of Taiwanese citizens $[7,8]$. In 1998, the Bureau of NHI drafted a prospective payment program to encourage integrated care for mechanically ventilated patients, which was implemented in July 2000 [9]. After several revisions, this program ultimately covered four types of mechanical ventilator care: ICU care (acute stage, $<21$ days), respiratory care center (RCC, a sub-acute stage for weaning training of up to 42 days), respiratory care ward ( $\mathrm{RCW}$, a chronic stage or long-term care), and home care service (stable stage, in which the patient is cared for directly by family caregivers).

Unlike patients on PMV in other countries [10-14], many patients in Taiwan suffer from concomitant cognitive impairments that preclude direct assessment of their subjective QOL, making evaluation by proxy unavoidable in many cases. The objective of this study was to evaluate the utility assessment of QOL in patients on PMV and to compare patients' own QOL assessments with those made on their behalf by proxies (family caregivers and professional nurses).

\section{Methods}

Subjects and methods

We recruited subjects from five institutions in northern and southern Taiwan and consecutively enrolled current PMV subjects who had already been on mechanical ventilation for at least for 21 days at various levels of care (ICU, RCC, and RCW): 1 medical center, 1 regional hospital, and 3 small local hospitals were included. The study began after approval was obtained from the Institutional Review Boards (IRBs) of the National Taiwan University Hospital and the Chia-Yi Christian Hospital. The three local hospitals also approved the study after reviewing the approval documents of the two major IRBs above. For subjects with basic cognition who were able to communicate (including through clear body language) and who scored at least 15 on the mini-mental status examination (MMSE) [15], we conducted QOL measurements using the EQ-5D in direct, face- to-face interviews. The primary caregivers (family members and nurses) were also asked to independently complete the EQ-5D questionnaire from the patient's point of view, i.e., each proxy rated how he or she thought the patient would rate his or her own QOL on the day of the interview. All the interviews with the patient and his/her proxy were required to be finished within 3 days, and we also made sure that the individual patient's clinical condition was stable by verifying with the medical records. The proxy's results were directly compared with the patient's own rating, which was considered to be the gold standard.

\section{Quality of life measured by EQ-5D}

The utility value of the QOL for patients on PMV was estimated using the EuroQol five-dimensional (EQ-5D) questionnaire, a generic preference-based instrument. The five dimensions assessed by the EQ-5D are mobility, selfcare, usual activities, pain/discomfort, and anxiety/depression, with three levels of severity (no problems, some/ moderate problems, and severe/extreme problems). This provides a utility value that ranges from 0 to 1 based upon the five-dimensional health state classification, in which 0 represents the worst health status and 1 represents perfect health [16, 17]. The EQ-5D instrument is a valid and reliable tool for measuring health status that has been extensively used in many countries as an outcome measure, including for critically ill patients [18-20]. It was also recently applied in Taiwan with good validity and moderate reliability [21]. We applied the value systems of the United States and the United Kingdom as a comparison $[17,22]$. There is general agreement that patients are the best raters of their QOL; however, when a patient's mental status is too poor, family caregivers and nurses may be considered as proxies [11-13]. QOL assessment by EQ-5D was conducted by three research assistants who were first trained in the standard operating procedure. For each patient and his/her proxy, the researcher recorded characteristics including age, gender, education, marital status, relationship to the patient, whether or not the proxy lived with the patient, frequency of caring for the patient, location (ICU or RCC or RCW), Glasgow Coma Scale (GCS), presence/absence of tracheotomy and duration of mechanical ventilation, and history of depression. The duration-to-date for each measurement was defined as beginning on the first day after PMV and ending on the date of interview by our researcher.

Statistical analysis

Binary and categorical variables were summarized using frequency counts and percentages. Continuous variables are presented as means if normally distributed. The agreement 
between QOL scores from patients, family caregivers, and nurses for each dimension was determined for each pair by calculating weighted kappa scores [23]. To evaluate any systematic tendency for proxy respondents to overestimate or underestimate QOL, we computed the patient-proxy and proxy-proxy mean differences in the final utility value of the EQ-5D. The mean difference between pair-scores and values were tested with the rank sum and paired $t$-tests to detect any significant difference when the sample size is small. We calculated the effect size by dividing the mean patient-proxy difference by the standard deviation of the patient responses. The magnitude of the standardized difference can be interpreted as follows: $|d|=0.2$, a small difference; $|d|=0.5$, a moderate difference; and $|d|=0.8$, a large difference [24]. The agreement between patients and proxies of the summary index of EQ-5D was assessed with one-way intra-class correlation coefficients (ICC) [25, 26]. An ICC or weighted kappa less than 0.4 was considered to indicate poor agreement, $0.40-0.75$ indicated moderate to good agreement, and values $>0.75$ indicated excellent agreement [23, 27-29]. Because the group was homogeneous in disease status and marginal distribution, the statistical ICC and weighted kappa underrate agreement [30]. Thus, we calculated the proportion of exact agreement, defined as the number of same response categories chosen between the index subject and the proxy divided by the total number of rating pairs [31].

\section{Results}

Basic characteristics of respondents

A total of 177 patients were invited for this study; 142 patients accepted interviews producing a response rate of $80 \%$, as summarized in Fig. 1. For the 55 patients able to assess their own QOL, we collected 44 patient-family caregiver pairs, 53 patient-nurse pairs, and 42 family caregiver-nurse pairs. We successfully collected 81 family caregiver-nurse pairs for 87 patients who were unable to assess their own QOL. We were unable to recruit any proxy for six patients with poor cognition.

The first proxy group (family caregivers) consisted of 129 persons; $57 \%$ were the patients' children, $58 \%$ lived with the patients, and $67 \%$ contacted the patients everyday. The second proxy group (nurses) consisted of 135 persons, $27 \%$ of whom were directly responsible for the patients' everyday care (Table 1).

Agreement in each dimension

Although the weighted kappa scores indicated poor to moderate agreement $(0.21-0.58)$ in the observable

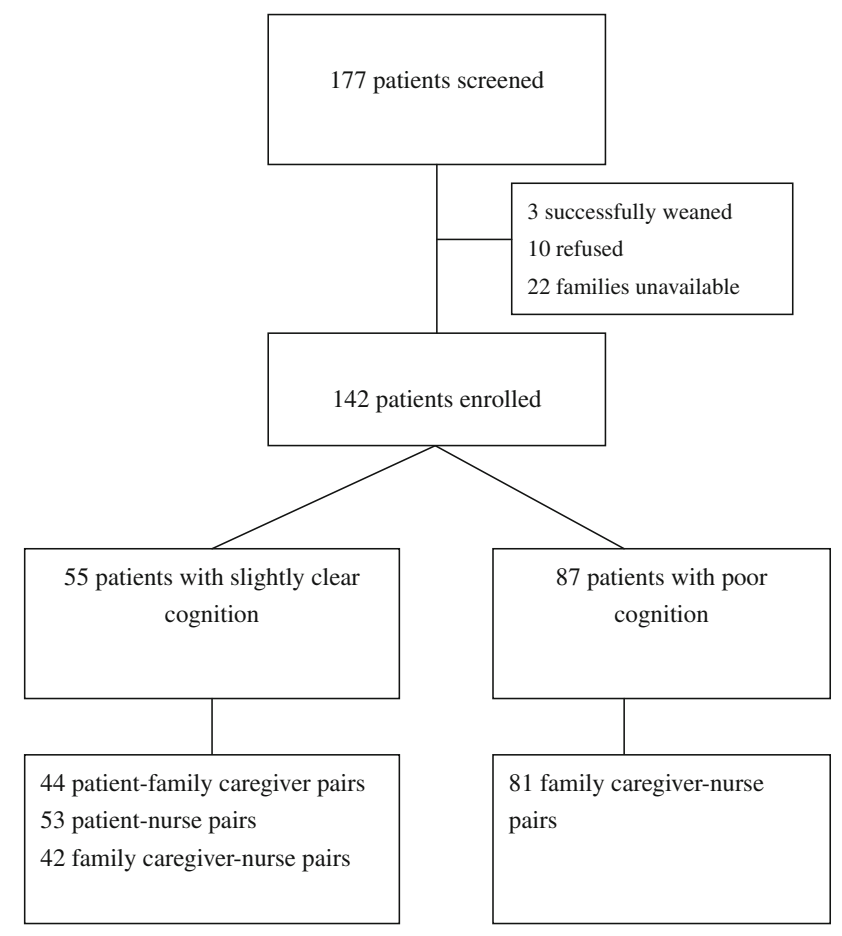

Fig. 1 Recruitment process for study participants

Table 1 Characteristics of study subjects and proxies (family caregivers and nurses)

\begin{tabular}{llll}
\hline Characteristics & Patients & $\begin{array}{l}\text { Family } \\
\text { caregivers }\end{array}$ & Nurses \\
\hline Total number of subjects & 55 & 129 & 135 \\
Mean age in years (SD) & $70.9(13.2)$ & $51.5(11.7)$ & 27.7 (3.6) \\
\% Females & 49 & 46 & 95 \\
\% Above junior high & 31 & 56 & 98 \\
school education & & & \\
\% Married & 96 & - & - \\
Relationship with the patient & & & \\
\% Spouse & - & 26 & - \\
\% Child & - & 57 & - \\
\% Others & - & 17 & - \\
\% Living together & - & 58 & - \\
\% Frequency of contact with patient & & \\
Daily & - & 67 & 27 \\
$>$ Once a week & - & 25 & 54 \\
$<$ Once a week & - & 8 & 19 \\
Glasgow Coma Scale (SD) & $9.9(2.5)$ & - & - \\
\% Tracheotomy & 71 & - & - \\
Duration of mechanical & $19.1(26.0)$ & - & \\
ventilation (months) & & & - \\
\hline
\end{tabular}

Values are expressed as means (standard deviations) or percentages

dimensions (mobility, self-care, and usual activities) and poor agreement $(0.00-0.35)$ in the subjective dimensions (pain/discomfort and anxiety/depression), the proportions 
Table 2 Frequency distribution of agreement among patients with fair to good cognition (mini-mental status examination score $>15$ ) for each dimension of the EQ-5D

\begin{tabular}{|c|c|c|c|c|c|}
\hline Dimension & $\begin{array}{l}\text { Patient } \\
n=55(\%)\end{array}$ & $\begin{array}{l}\text { Family caregiver } \\
n=44(\%)\end{array}$ & $\begin{array}{l}\text { Nurse } \\
n=53(\%)\end{array}$ & $\begin{array}{l}\text { Patient-family caregiver } n=44 \\
\text { weighted kappa (confidence intervals) } \\
\text { and [proportion of exact agreement] }\end{array}$ & $\begin{array}{l}\text { Patient-nurse } n=53 \text { weighted } \\
\text { kappa (confidence intervals) and } \\
\text { [proportion of exact agreement] }\end{array}$ \\
\hline \multicolumn{6}{|l|}{ Mobility } \\
\hline No problems & 0 & 0 & $2(4)$ & & \\
\hline Problems & $11(20)$ & $8(18)$ & $10(19)$ & $0.58(0.28-0.88)$ & $0.28(0.01-0.55)$ \\
\hline Confined to bed & $44(80)$ & $36(82)$ & $41(77)$ & {$[86 \%]$} & {$[75 \%]$} \\
\hline \multicolumn{6}{|l|}{ Self-care } \\
\hline No problems & 0 & 0 & 0 & & \\
\hline Some problems & $8(15)$ & $3(7)$ & $11(21)$ & $0.56(0.19-0.93)$ & $0.21(-0.11-0.52)$ \\
\hline Unable to wash/dress & $47(85)$ & $41(93)$ & $42(79)$ & [91\%] & {$[77 \%]$} \\
\hline \multicolumn{6}{|l|}{ Usual activities } \\
\hline No problems & 0 & 0 & $1(2)$ & & \\
\hline Some problems & $11(20)$ & $7(16)$ & $11(21)$ & $0.44(0.09-0.79)$ & $0.26(-0.03-0.56)$ \\
\hline Unable to perform & $44(80)$ & 37 (84) & $41(77)$ & [91\%] & {$[75 \%]$} \\
\hline \multicolumn{6}{|l|}{ Pain/discomfort } \\
\hline None & $20(36)$ & $10(23)$ & $8(15)$ & & \\
\hline Moderate & $17(31)$ & $19(43)$ & $38(72)$ & $0.35(0.13-0.57)$ & $0.00(-0.17-0.16)$ \\
\hline Extreme & $18(33)$ & $15(34)$ & $7(13)$ & {$[52 \%]$} & {$[28 \%]$} \\
\hline \multicolumn{6}{|l|}{ Anxiety/depression } \\
\hline None & $27(50)$ & $13(30)$ & $7(13)$ & & \\
\hline Moderate & $14(25)$ & $13(30)$ & $36(68)$ & $0.35(0.13-0.57)$ & $0.18(0.01-0.34)$ \\
\hline Extreme & $14(25)$ & $18(40)$ & 10 (19) & [52\%] & [38\%] \\
\hline
\end{tabular}

of exact agreement were more than 75 and $28 \%$ for observable and subjective dimensions, respectively, indicating high agreement on the former dimension for both family caregivers and nurses (Table 2). Family caregivers in particular displayed better agreement with the patients compared to nurses on subjective feelings of pain/discomfort and anxiety/depression. In patients with poor cognition, the proportions of exact agreement of family caregiver-nurse pairs were all above $98 \%$ in the observable dimensions but only $37-38 \%$ in the subjective dimensions (Table 3).

\section{Agreement of EQ-5D index}

The mean EQ-5D index among the 55 patients was $0.23 \pm 0.20$. The mean differences in the EQ-5D index were $-0.03 \pm 0.14$ and $0.03 \pm 0.22$ for patient-family caregiver pairs and patient-nurse pairs, respectively; this difference was not statistically significant (Table 4). In PMV patients with poor cognition, the average EQ-5D index assigned by family caregivers and nurses were $0.12 \pm 0.10(0-0.44)$ and $0.14 \pm 0.11(0-0.63)$, respectively. The effect size of the mean differences for these measurements was 0.13 , which is small [24]. The ICC of the EQ-5D index for patient-family caregiver pairs was excellent (0.76), which seems much higher than that of the patient-nurse pairs (0.36) (Table 4).

\section{Discussion}

To our knowledge, this study is the first that validates agreement on QOL between PMV patients and their proxies (family caregivers and nurses) as measured by the EQ-5D preference instrument; in addition, these conclusions were validated in both PMV patients with good and poor cognition. Our findings demonstrate that family caregivers tended to rate all dimensions as being more severe than did patients themselves and that family caregivers' assessments were in closer agreement with patients' assessments than were those of their nurses, using either observable dimensions or subjective feelings. Moreover, agreement on the observable dimensions between family caregivers and nurses was higher (proportions of exact agreement $=98-99 \%)$ in PMV patients with poor cognition.

Our study corroborates previous investigations finding better agreement between patients and their proxies for directly observable dimensions (mobility, self-care, and usual activities) than for assessments of subjective feelings 
Table 3 Frequency distribution of agreement among patients with poor cognition and their proxies for each dimension in the EQ-5D

\begin{tabular}{|c|c|c|c|}
\hline Dimension & $\begin{array}{l}\text { Family } \\
\text { caregiver } \\
n=81(\%)\end{array}$ & $\begin{array}{l}\text { Nurse } \\
n=81(\%)\end{array}$ & $\begin{array}{l}\text { Family caregiver-nurse } n=81 \\
\text { weighted kappa (confidence intervals) } \\
\text { and [proportion of exact agreement] }\end{array}$ \\
\hline \multicolumn{4}{|l|}{ Mobility } \\
\hline No problems & 0 & 0 & \\
\hline Problems & $1(1)$ & $1(1)$ & $-0.01(-0.03-0.00)$ \\
\hline Confined to bed & $80(99)$ & $80(99)$ & {$[98 \%]$} \\
\hline \multicolumn{4}{|l|}{ Self-care } \\
\hline No problems & 0 & 0 & \\
\hline Some problems & 0 & $1(1)$ & $0.00(-0.00-0.00)$ \\
\hline Unable to wash/dress & $81(100)$ & 80 (99) & {$[99 \%]$} \\
\hline \multicolumn{4}{|l|}{ Usual activities } \\
\hline No problems & 0 & 0 & \\
\hline Some problems & 0 & $1(1)$ & $0.00(-0.00-0.00)$ \\
\hline Unable to perform & $81(100)$ & 80 (99) & {$[99 \%]$} \\
\hline \multicolumn{4}{|l|}{ Pain/discomfort } \\
\hline None & $16(20)$ & $9(11)$ & \\
\hline Moderate & $27(33)$ & $45(56)$ & $0.05(-0.13-0.23)$ \\
\hline Extreme & $38(47)$ & $27(33)$ & {$[37 \%]$} \\
\hline \multicolumn{4}{|l|}{ Anxiety/depression } \\
\hline None & $19(23)$ & $10(12)$ & \\
\hline Moderate & $30(37)$ & $48(59)$ & $0.08(-0.08-0.24)$ \\
\hline Extreme & $32(40)$ & $23(28)$ & {$[38 \%]$} \\
\hline
\end{tabular}

Table 4 Differences and reliabilities of EQ-5D index measured in patients on PMV (prolonged mechanical ventilation) and their proxies, according to value systems established in the United Kingdom (UK) and United States (US)

\begin{tabular}{|c|c|c|c|c|c|}
\hline & \multicolumn{2}{|c|}{ Mean difference ${ }^{\mathrm{ab}} \pm \mathrm{SD}$ (minimum-maximum) } & \multicolumn{2}{|c|}{ Effect size } & \multirow{2}{*}{$\begin{array}{l}\text { ICC }^{\text {cd }} \text { (confidence } \\
\text { intervals) }\end{array}$} \\
\hline & US & UK & US & UK & \\
\hline \multicolumn{6}{|c|}{ Patients with partial cognition capable of responding (mini-mental status examination, MMSE $\geqq 15$ ) } \\
\hline Patient-family caregiver $(n=44)$ & $-0.03 \pm 0.14(-0.52-0.22)$ & $-0.04 \pm 0.17-0.39-0.37)$ & 0.21 & 0.24 & $0.76(0.60-0.86)$ \\
\hline Patient-nurse $(n=53)$ & $0.03 \pm 0.22(-0.60-0.50)$ & $0.00 \pm 0.23(-0.51-0.46)$ & 0.14 & 0.00 & $0.36(0.11-0.58)$ \\
\hline Family caregiver-nurse $(n=42)$ & $0.07 \pm 0.18 *(-0.38-0.40)$ & $0.07 \pm 0.22 *(-0.44-0.50)$ & 0.39 & 0.32 & $0.49(0.22-0.69)$ \\
\hline \multicolumn{6}{|c|}{ Patients with poor cognition and unable to respond (MMSE $<15$ ) } \\
\hline Family caregiver-nurse $(n=81)$ & $0.02 \pm 0.15(-0.38-0.52)$ & $0.02 \pm 0.20(-0.37-0.44)$ & 0.13 & 0.10 & $0.00(-0.22-0.20)$ \\
\hline
\end{tabular}

$* p<0.05$

${ }^{a}$ Proxy minus patient score: a negative sign indicates that proxies underestimated the score

${ }^{\mathrm{b}}$ Nurse minus family caregiver: a negative sign indicates that the nurses consistently scored lower than did family caregivers

c ICC: intra-class correlation coefficients

${ }^{\mathrm{d}}$ US value system only

and mental health (pain/discomfort and anxiety/depression) [32-34]. However, family caregivers tended to rate all dimensions at a slightly higher severity than did patients themselves, while nurses gave less severe ratings compared to patients (Table 2). Family caregivers were generally allowed to stay with patients after their hospitalization in RCW institutions, which may partially explain why they were apparently able to perceive so accurately the feelings of PMV patients, with all proportions of the exact agreement above $86 \%$ for observable dimensions and $52 \%$ for subjective dimensions. In addition, no significant differences were identified between the final EQ-5D utility values from the patient-family caregiver pairs (Table 4). In contrast, only $27 \%$ of the interviewed nurses had daily contact with the patients, as they generally rotated and took days off each week (Table 1). We applied multiple regression analysis to estimate the potential effect of this determinant on the mean difference between patient-nurse 
pairs, but it did not show any statistical significance. Overall, the agreement within patient-family caregiver pairs is consistently closer than that of patient-nurse pairs, indicating that family caregivers may be a more suitable proxy for PMV patients in the Taiwanese culture.

In PMV patients with poor cognition, it is not surprising that both the weighted kappa (Table 3) and ICC (Table 4) were very low, because these patients were generally bedridden in $\mathrm{RCC} / \mathrm{RCW}$ units, and there is almost zero variation in the observable dimensions of mobility, self-care, and usual activities. Thus, such extremely limited distributions hamper the values of weighted kappa and ICC, and we must also provide the results of proportions of exact agreement, which were as high as $37-99 \%$ (Table 3) to avoid misinterpretation.

Nonetheless, there was no statistically significant difference between the final utility values of EQ-5D from patientfamily caregiver and patient-nurse pairs in PMV patients with partial cognition. Because the EQ-5D value system of Taiwan has not yet been published, we applied the values obtained from the United States and United Kingdom for sensitivity analysis $[17,22]$. Although the values based on the US system seem smaller than those from the United Kingdom, they yielded similar results, apparently due to the limited variation of health states in these patients (Table 4). Thus, the nurse's rating appears as acceptable as that of a family caregiver for use as a proxy to rate the utility value of the EQ-5D in PMV patients with poor cognition, even though the ICC of these pairs was only 0.00 .

A possible limitation of this study was its cross-sectional design. Although we conducted a multiple regression analysis using the utility value of EQ-5D as the dependent variable and the duration of mechanical ventilation as an independent variable in the model, there was no significant difference. Thus, we were unable to answer whether the agreement of QOL measurements between patients and proxies may change longitudinally over different time points. Another possible limitation was the $80 \%$ response rate and the degree to which our sample was representative of the population as a whole. There was no statistically significant difference in patient characteristics between respondents and non-respondents. However, because family members of 22 of the 35 non-respondents did not come to visit the patients during our 3-4 week study period, those patients might have a poorer QOL compared to that of the others. Ten family caregivers refused to be interviewed at the time of our request, as they were coping with their relative's deteriorating condition. Thus, our results might have overestimated the QOL of PMV patients. Finally, the extremely skewed responses in the objective dimensions of the EQ-5D in patients with poor cognition result in a generally low weighted kappa (Table 3), reflecting the inappropriateness of using such indicators.
Thus, we have calculated the proportion of exact agreement to allow us to make a suitable inference.

\section{Conclusions}

Although the sensitivity analysis did not show any significant differences in overall utility value of QOL measurements using EQ-5D between patients on PMV and their proxies, family caregivers exhibited closer agreement with the patients than did nurses on QOL evaluation, especially on rating subjective feelings of pain/discomfort and anxiety/depression. However, there was no difference between the two proxies in the final utility values for PMV patients with poor cognition. Further research into these issues is still needed in subjects from different cultures to corroborate our findings.

Acknowledgments This work was partially supported by grants from the National Health Research Institute (NHRI, Grant 97A1HDPP01-014 and 98A1-PHAPHD-014), Chia-Yi Christian Hospital (CYCH, Grant R98-3), and the National Science Council (NSC, Grant 98-2341-B-002-129) in Taiwan. The authors also thank the members of the respiratory care ward of Chia-Yi Hospital, the Department of Health, Executive Yuan, Taiwan; the private Chen Ren-Der Hospital; Yang Ming Hospital (Chia-Yi); and the National Taiwan University Hospital for helping us to recruit study subjects.

\section{References}

1. Carson, S. S. (2006). Outcomes of prolonged mechanical ventilation. Current Opinion in Critical Care, 12(5), 405-411. doi: 10.1097/01.ccx.0000244118.08753.dc.

2. Carson, S. S., Cox, C. E., Holmes, G. M., \& Howard, A. (2006). The changing epidemiology of mechanical ventilation: A population-based study. Journal of Intensive Care Medicine, 21(3), 173-182. doi:10.1177/0885066605282784.

3. Cox, C. E., Carson, S. S., Holmes, G. M., \& Howard, A. (2004). Increase in tracheotomy for prolonged mechanical ventilation in North Carolina, 1993-2002. Critical Care Medicine, 32(11), 2219-2226. doi:10.1097/01.CCM.0000145232.46143.4.

4. Douglas, S. L., Daly, B. J., Gordon, N., \& Brennan, P. F. (2002). Survival and quality of life: Short term versus long-term ventilator patients. Critical Care Medicine, 30, 2655-2662. doi: 10.1097/01.CCM.0000034688.55283.6.

5. Combes, A., Costa, M. A., Trouillet, J. L., Baudot, J., Mokhtari, M., Gibert, C., et al. (2003). Morbidity, mortality, and quality-oflife outcomes of patients requiring $\geqq 14$ days of mechanical ventilation. Critical Care Medicine, 31, 1373-1381. doi:10.1097/ 01.CCM.0000065188.87029.C3.

6. Chatila, W., Kreimer, D. T., \& Criner, G. J. (2001). Quality of life in survivors of prolonged mechanical ventilatory support. Critical Care Medicine, 29(4), 737-742. Retrieved from http://journals. lww.com/ccmjournal/pages/default.aspx.

7. Department of Statistics, Ministry of the Interior (2009). Number of population. Available online at: http://www.moi.gov.tw/stat/ english/monthly.asp. Accessed August 24, 2009.

8. Bureau of National Health Insurance (2009). Insurance coverage. Available online at: http://www.nhi.gov.tw/webdata. Accessed August 24, 2009. 
9. Bureau of National Health Insurance (2009). Prospective payment system of ventilator dependents managed care demonstration. Available online at. http://www.nhi.gov.tw/webdata. (In Chinese). Accessed August 24, 2009.

10. Coucill, W., Bryan, S., Bentham, P., Buckley, A., \& Laight, A. (2001). EQ-5D in patients with dementia: An investigation of inter-rater agreement. Medical Care, 39(8), 760-771. Retrieved from http://journals.lww.com/lww-medicalcare/pages/default.aspx.

11. Bryan, S., Hardyman, W., Bentham, P., Buckley, A., \& Laight, A. (2005). Proxy completion of EQ-5D in patients with dementia. Quality of Life Research, 14(1), 107-118. doi:10.1007/s11136004-1920-6.

12. Pickard, A. S., \& Knight, S. J. (2005). Proxy evaluation of healthrelated quality of life: A conceptual framework for understanding multiple proxy perspectives. Medical Care, 43(5), 493-499. Retrieved from http://journals.lww.com/lww-medicalcare/pages/ default.aspx.

13. McPhail, S., Beller, E., \& Haines, T. (2008). Two perspectives of proxy reporting of health-related quality of life using the Euroqol-5D, an investigation of agreement. Medical Care, 46(11), 1140-1148. doi:10.1097/MLR.0b013e31817d69a6.

14. Sneeuw, K. C. A., Sprangers, M. A. G., \& Aaronson, N. K. (2002). . The role of health care providers and significant others in evaluating the quality of life of patients with chronic disease. Journal of Clinical Epidemiology, 55(11), 1130-1143. Retrieved from http://www.sciencedirect.com/science/journal/08954356.

15. Folstein, M. F., Folstein, S. E., \& McHugh, P. R. (1975). "Minimental state". A practical method for grading the cognitive state of patients for the clinician. Journal of Psychiatric Research, 12(3), 189-198. Retrieved from http://www.sciencedirect.com/ science/journal/00223956.

16. EuroQol Group (1990). EuroQol-a new facility for the measurement of health-related quality of life. Health Policy, 16(3), 199-208. Retrieved from http://www.sciencedirect.com/science/ journal/01688510.

17. Szende, A., Oppe, M., \& Devlin, N. (2007). EQ-5D value sets: Inventory, comparative review and user guide. UK: EuroQol Group.

18. Badia, X., Diaz-Prieto, A., Rue, M., \& Patrick, D. L. (1996). Measuring health and health state preferences among critically ill patients. Intensive Care Medicine, 22(12), 1379-1384. doi: $10.1007 / \mathrm{s} 001340050267$.

19. Cristina, G., Armando, T. P., \& Altamiro, C. P. (2002). Quality of life after intensive care-evaluation with EQ-5D questionnaire. Intensive Care Medicine, 28(7), 898-907. doi:10.1007/s00134002-1345-z.

20. Granja, C., Dias, C., Costa-Pereira, A., \& Sarmento, A. (2004). Quality of life of survivors from severe sepsis and septic shock may be similar to that of others who survive critical illness. Critical Care, 8, R91-R98. Retrieved from http://journals.lww. com/lww-medicalcare/pages/default.aspx.
21. Chang, T. J., Tarn, Y. H., Hsieh, C. L., Liou, W. S., Shaw, J. W., \& Chiou, X. G. (2007). Taiwanese version of the EQ-5D: Validation in a representative sample of the Taiwanese population. Journal of the Formosan Medical Association, 106(12), 10231031. Retrieved from http://fma.mc.ntu.edu.tw/.

22. Shaw, J. W., Johnson, J. A., \& Coons, S.J. (2007). US valuation of the EQ-5D health states: Development and testing of the D1 valuation model. Med Care, 43(3), 203-220. Retrieved from http://journals.lww.com/lww-medicalcare/pages/default.aspx.

23. Altman, D. G. (1991). Practical statistics for medical research (1st ed.). London: Chapman and Hall.

24. Cohen, J. (1988). Statistical power analysis for the behavior sciences (2nd ed.). New Jersey: Lawrence Erlbaum Associates.

25. Deyo, R. A., Diehr, P., \& Patrick, D. L. (1991). Reproducibility and responsiveness of health status measures. Statistics and strategies for evaluation. Controlled Clinical Trials, 12(4Suppl), 142S-158S. Retrieved from http://www.sciencedirect.com/ science/journal/01972456.

26. Schrout, P., \& Fleiss, J. (1979). Intraclass correlations: Uses in assessing rater reliability. Psychiatric Bulletin, 86(2), 420-428. Retrieved from http://pb.rcpsych.org/.

27. Nunnally, J. C. (1994). Psychometric theory. New York: McGraw-Hill.

28. Lee, J., Koh, D., \& Ong, C. N. (1989). Statistical evaluation of agreement between two methods for measuring a quantitative variable. Computers in Biology \& Medicine, 19(1), 61-70. Retrieved from http://www.sciencedirect.com/science/journal/00104825.

29. Fleiss, J. L. (1986). The design and analysis of clinical experiments. New York: Wiley.

30. Agresti, A., \& Ghosh, A. (1995). Rank kappa: Describing potential impact of marginal distributions on measures of agreement. Biometrical Journal, 37(7), 811-820. doi:10.1002/ bimj.4710370705.

31. Novella, J. L., Jochum, C., Jolly, D., et al. (2004). Agreement between patients' and proxies' reports of quality of life in Alzheimer's disease. Quality of Life Research, 10(5), 443-452. doi: 10.1023/A:1012522013817.

32. Tamim, H., McCusker, J., \& Dendukuri, N. (2002). Proxy reporting of quality of life using the EQ-5D. Medical Care, 40(12), 1186-1195. Retrieved from http://journals.lww.com/ lww-medicalcare/pages/default.aspx.

33. Pickard, A. S., Johnson, J. A., Feeny, D. H., Shuaib, A., Carriere, K. C., \& Nasser, A. M. (2004). Agreement between patient and proxy assessments of health-related quality of life after stroke using the EQ-5D and health utilities index. Stroke, 35(2), 607612. doi:10.1161/01.STR.0000110984.91157.BD.

34. Andresen, E. M., Vahle, V. J., \& Lollar, D. (2001). Proxy reliability: Health-related quality of life (HRQoL) measures for people with disability. Quality of Life Research, 10, 609-619. doi:10.1023/A:1013187903591. 